\title{
The war against influenza: discovery and development of sialidase inhibitors
}

\section{Mark von Itzstein}

Abstract | The threat of a major human influenza pandemic, in particular from highly aggressive strains such as avian $\mathrm{H} 5 \mathrm{~N} 1$, has emphasized the need for therapeutic strategies to combat these pathogens. At present, two inhibitors of sialidase (also known as neuraminidase), a viral enzyme that has a key role in the life cycle of influenza viruses, would be the mainstay of pharmacological strategies in the event of such a pandemic. This article provides a historical perspective on the discovery and development of these drugs - zanamivir and oseltamivir — and highlights the value of structure-based drug design in this process.

The emergence of the extremely aggressive avian $\mathrm{H} 5 \mathrm{~N} 1$ influenza virus, in particular in Asia, has made the likelihood of a human influenza pandemic and the possible socioeconomic impact a major worldwide concern ${ }^{1-3}$. The appearance of $\mathrm{H} 5 \mathrm{~N} 1$, and the human fatalities it has already caused, has heightened the awareness of both the general population and governments to the threat of influenza virus to the extent that many governments have implemented preparedness plans, and available anti-influenza drugs are being stockpiled.

Historically, the first drugs available for the treatment of influenza were the adamantane-based M2 ion channel protein inhibitors, rimantidine and amantadine $e^{4,5}$. These compounds have only been useful in the treatment of influenza A infection, because only the A strains of the virus have M2 ion channel proteins ${ }^{4-7}$. Although both drugs can be effective against influenza virus A infection, they have been reported to cause CNS side effects ${ }^{5,6}$, and have given rise to the rapid emergence of drug-resistant viral strains ${ }^{7}$.

Given such issues, there has been considerable effort worldwide to discover novel therapeutic agents against all types of influenza, and several valuable reviews concerned with aspects of influenza virus have been published (for example, REFS 8-11). Here, after providing some background on the influenza virus and its key surface glycoproteins, this article describes the discovery and development of influenza virus sialidase (also known as neuraminidase/exo- $\alpha$-sialidase; EC 3.2.1.18) inhibitors, which are now at the forefront of defences against a flu pandemic. Focused efforts to develop such drugs using structural information began in the 1980s, and provide one of the earliest examples of the application of structurebased drug design.

\section{The virus}

Influenza virus belongs to the orthomyxoviridae family, which is subdivided into three serologically distinct types: A, B and C. Only influenza virus $A$ and $B$ appear to be of concern as human pathogens as influenza $C$ virus does not seem to cause significant disease ${ }^{12,13}$. Further classification of influenza virus is based on the antigenic properties of its surface glycoproteins haemagglutinin and sialidase ${ }^{14}$ (FIG. 1), both of which are essential for infection to proceed (FIG. 2). These surface glycoproteins are carbohydrate-recognizing proteins and in

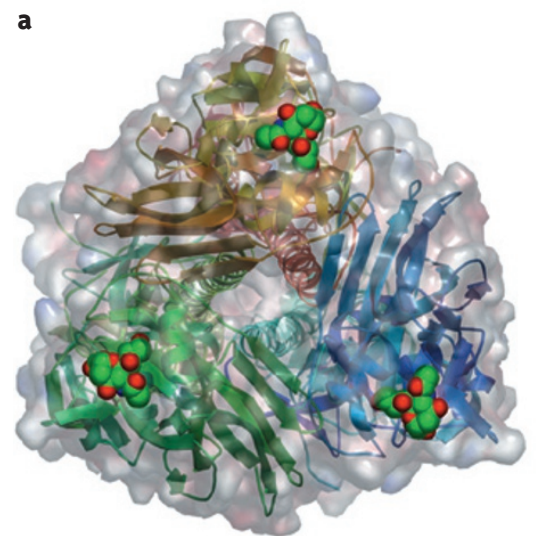

Figure 1 | Influenza virus surface glycoproteins. a | A view of the influenza virus haemagglutinin trimer complexed with $\mathrm{N}$-acetylneuraminic acid (Neu5Ac; in CPK form). $\mathbf{b} \mid \mathrm{A}$ monomeric subunit of influenza A virus sialidase complexed with Neu5Ac (in CPK form). The catalytic site is located near the pseudo-symmetry axis.

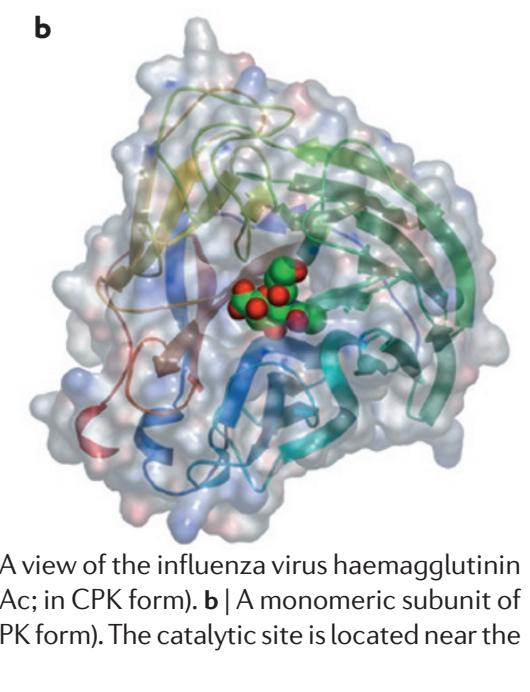

humans are known to recognize the sialic acid $N$-acetylneuraminic acid (Neu5Ac; which has $\alpha$ and $\beta$ forms when not conjugated; compound 1, FIC. 3), which is typically associated as the $\alpha$-linked terminal carbohydrate unit of upper respiratory tract and lung-associated glycoconjugates ${ }^{13,15}$.

Haemagglutinin is made up of three identical subunits (FIG. 1a) and is anchored to the lipid membrane of the virus ${ }^{13}$. This glycoprotein seems to have two significant roles. The first is to provide an initial point of contact for the virus to the target host cell-surface glycoconjugates by $\alpha$-ketosidically linked terminal Neu5Ac residues ${ }^{10,16,17}$. The second is to trigger the internalization process of the virus through fusion of the viral envelope with the host cell ${ }^{10,18}$. A multitude of influenza virus haemagglutinin structures have been determined, as well as the structures of several haemagglutinin-ligand complexes ${ }^{19-22}$.

Influenza virus sialidase is an enzyme made up of four identical subunits that is also anchored to the viral membrane ${ }^{23}$. The enzyme is an exoglycohydrolase and cleaves $\alpha$-ketosidically linked Neu5Ac residues that cap the ends of various glycoconjugates ${ }^{11}$. The significance of the sialidase action is that it assists in the movement of virus particles through the upper respiratory tract as well as in the release of virion progeny from infected cells ${ }^{24,25}$. Several influenza virus sialidase crystal structures, including a number of complexes of influenza virus sialidase with Neu5Ac (FIG. 1 b) and its derivatives, have been determined ${ }^{26-28}$.

The essential roles, although controversial at times, played by both of these surface glycoproteins in the infectious life cycle of the virus (FIG. 2), coupled with a significant 


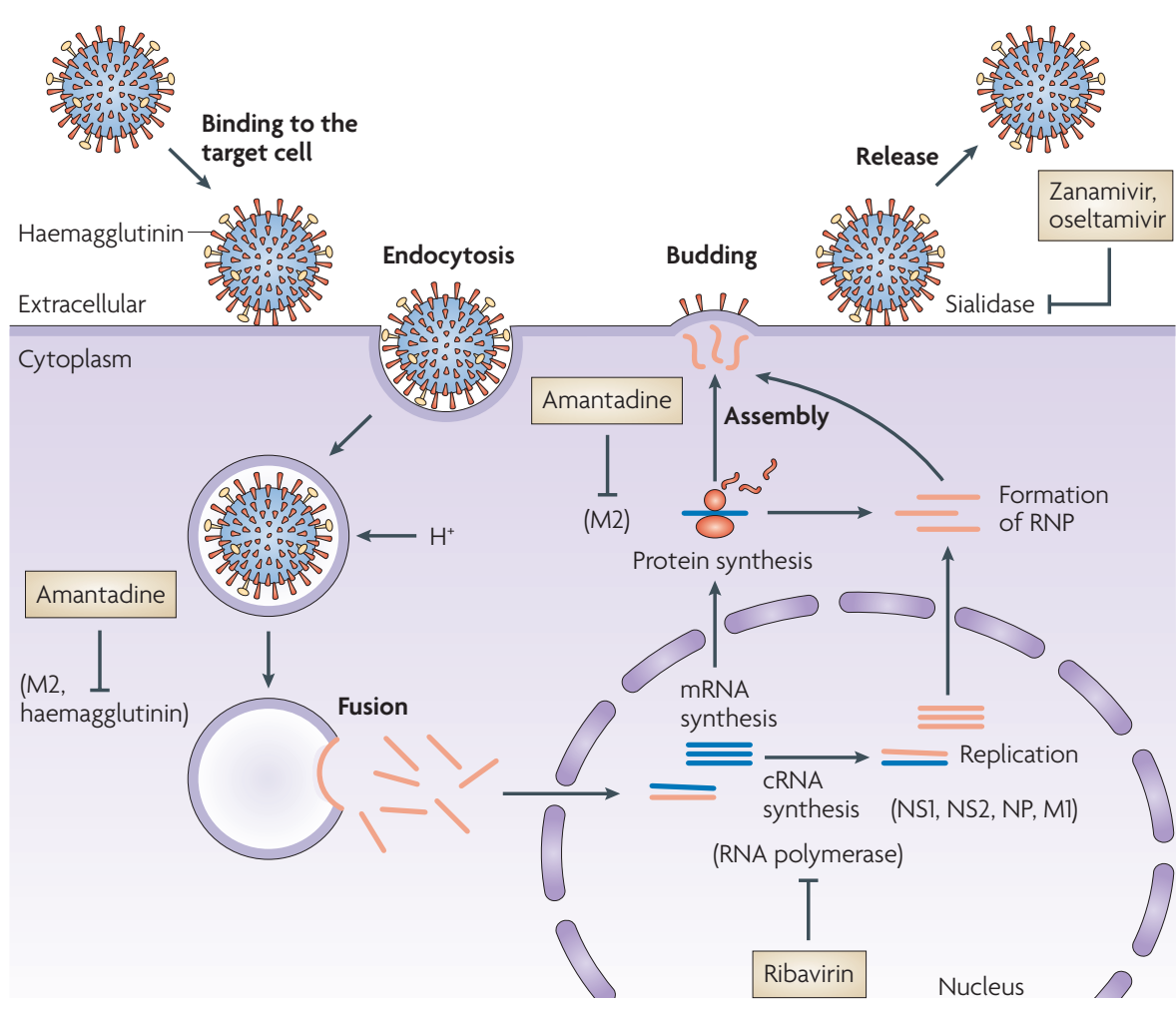

Figure 2 | Life cycle of the influenza virus and targets for therapeutic intervention. The surface of influenza virus $A$ is decorated with three proteins: an $\mathrm{M} 2$ ion channel protein, the lectin haemagglutinin and the enzyme sialidase. Typically, the influenza virus adheres to the target host cell by using its surface glycoprotein haemagglutinin to recognize glycoconjugates such as GD1a that display terminal $\alpha$-linked $N$-acetylneuraminic acid ( $\alpha$-Neu5Ac, compound 1a, FIG. 3) residues. The virus is then endocytosed, fusion occurs and the host-cell machinery is engaged to produce the necessary viral components. Subsequent viral protein synthesis and particle assembly in the host cell prepares the virion progeny for the budding process to exit the host cell. The enzyme sialidase cleaves the terminal $\alpha-N e u 5 A c$ residues from both the newly synthesized virion progeny glycoproteins as well as from the host-cell surface. The action of sialidase enables the host-cell-surface aggregated virion progeny to elute away from the infected cell and seek new host cells to infect. Both haemagglutinin and sialidase have been proposed as potential anti-influenza drug discovery targets. As described in the main text, zanamivir and oseltamivir efficiently block the action of sialidase and significantly inhibit the release mechanism. The M2 ion channel protein of influenza virus A has also been targeted by a class of drugs referred to as the adamantanes, which include amantadine and rimantadine. Last, ribavirin has also been demonstrated to inhibit virus replication by acting on the RNA polymerase function.

body of available structural information, have provided exciting opportunities for rational structure-based discovery of antiinfluenza agents ${ }^{11,29}$. The most successful structure-based anti-influenza drug discovery programme has arisen from targeting the sialidase function. This article describes aspects of the discovery of the first potent designed influenza virus sialidase inhibitor - and now commercially available inhaled anti-influenza drug - zanamivir (Relenza; GlaxoSmithKline $)^{30}$, and the discovery of the subsequently approved orally bioavailable drug oseltamivir (Tamiflu; Gilead/Roche) ${ }^{31}$. More recent approaches to discover nextgeneration sialidase inhibitors to combat influenza infection, particularly pandemic influenza, are also discussed. nucleophilic attack of a strain-independent highly conserved tyrosine residue. This catalytic intermediate is subsequently hydrolytically released as $\alpha-\mathrm{Neu} 5 \mathrm{Ac}^{33}$ (compound 1a, FIG. 3).

Several high-resolution X-ray crystal structures of sialidase complexed with various small-molecule inhibitors have been determined, including Neu5Ac (FIG. 4a). Most strikingly, the active site consists of a number of distinct adjoining pockets that are lined by eight highly conserved amino-acid residues that make direct contact with Neu5Ac and its derivatives ${ }^{27}$ (FIG. 4b). In addition, there are a further ten amino-acid residues invariant in all strains of influenza virus within the vicinity of the active site that appear to be important primarily in the stabilization of the architecture of the active site ${ }^{27,37}$.

Upon binding, Neu5Ac-containing glycoconjugates are oriented in the active site through interaction with a cluster of three arginine residues and the Neu5Ac moiety makes a number of significant contacts with active-site residues $^{38,39}$ (FIG. 4C). Specifically, further orientation of the Neu5Ac moiety is facilitated by several additional interactions within the active site, including hydrogen bonding of the $\mathrm{C}-5$ acetamido group carbonyl oxygen to Arg 152 and its $\mathrm{N}-\mathrm{H}$ to a buried water molecule. Favourable hydrophobic contacts to residues Trp178 and Ile222 are also made by the methyl of the C- 5 acetamido group. Additional hydrogen-bond networks are formed by the C- 8 and C-9 hydroxyl groups of the glycerol side chain to the carboxylate oxygens of residue Glu276, while the C-4 hydroxyl group associates with the carboxylate oxygen of Glu119. All of the amino-acid residues mentioned above are fully conserved across the natural strains of influenza virus known so far ${ }^{23,37}$.

\section{Structure-based inhibitor design}

Although several influenza virus sialidase inhibitors had been reported in the literature before structural information was available, none had shown efficacy in an in vivo model (for reviews, see REFS 11,29,40). From these studies, the unsaturated Neu5Ac derivative 2-deoxy-2,3-didehydro- $N$-acetylneuraminic acid (Neu5Ac2en; compound 2a, FIG. 3), a micromolar inhibitor of influenza virus sialidase, has provided the most potent inhibitor core template.

The first sialidase crystals were successfully grown from purified protein and subsequently, more suitable crystals were obtained for diffraction purposes and resulted in the X-ray crystal structure determination of influenza virus sialidase ${ }^{26,27,37}$. This structural 
information proved valuable in the discovery and development of zanamivir and oseltamavir, as described below.

The discovery and development of zanamivir. The initial crystal structure ${ }^{26,27}$ of influenza virus sialidase had a resolution of $\sim 3.0 \AA$, and consequently had limited value in structurebased drug design. Therefore, an initial focus in the discovery of influenza virus sialidase inhibitors was on substrate-like Neu5Ac derivatives, particularly 2 -deoxy- $\alpha-\mathrm{D}-N$ acetylneuraminic acid (2-deoxy- $\alpha$-Neu5Ac) derivatives (compound 3, FIG. 3). Based on an understanding of the enzyme's catalytic mechanism ${ }^{33,40}$, it was thought that such compounds might not be rapidly metabolized and should be recognized by the enzyme as a result of the compound's substrate/productlike characteristics ${ }^{41}$.

The parent unsubstituted template of this series, 2-deoxy- $\alpha$-Neu5Ac (compound 3, FIG. 3), was the first compound produced by the von Itzstein group to be evaluated in vivo in a mouse model of influenza infection by Glaxo researchers led by Charles Penn and Janet Cameron. This compound showed weak, but measurable, effects in animals infected with influenza virus. It was intriguing that 2 -deoxy- $\alpha$-Neu 5 Ac, but not the unsaturated $\mathrm{N}$-trifluoroacetylated derivative of Neu5Ac (compound 2b, FIG. 3; the most potent influenza virus sialidase inhibitor up until the late $1980 \mathrm{~s}^{42-45}$ ) had in vivo, albeit weak, activity. The general view was that compound $2 \mathrm{~b}$ may have suffered from either metabolism ${ }^{44}$ or rapid clearance, as demonstrated by others for the parent template, Neu5Ac2en (compound 2a, FIG. 3$)^{45}$. To better understand this interesting observation, Penn, Cameron and colleagues re-evaluated Neu5Ac2en in their established mouse model under identical conditions to those used for 2-deoxy- $\alpha$-Neu5Ac, and it demonstrated good activity in the mouse model experiments.

On the basis of these experiments, the choice of carbohydrate template was changed. Concomitantly, further refinement of a number of sialidase crystal structures (BOX 1) in complex with Neu5Ac and the now confirmed in vivo inhibitor, Neu5Ac2en, were successfully completed. This improvement in structural resolution enabled the commencement of a fully fledged structure-based drug design effort based on these X-ray crystal structures. Computational chemistry techniques were used to probe the active site of influenza virus sialidase in an attempt to design structurally modified Neu5Ac2en derivatives that might be more potent inhibitors ${ }^{30,39,46}$.
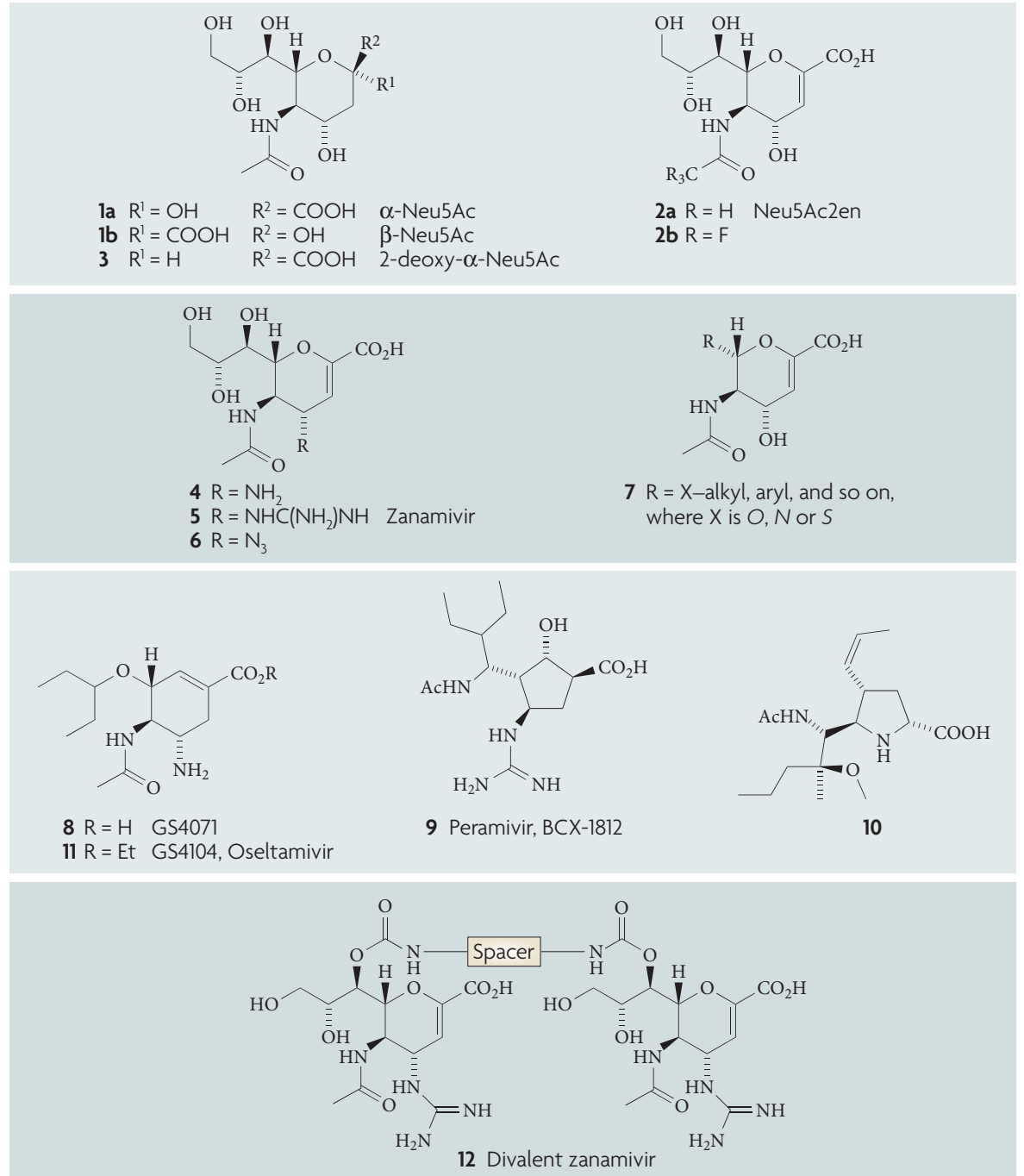

Figure 3 | Chemical structures of selected compounds. 1a, $\alpha-N$-acetylneuraminic acid ( $\alpha$-Neu5Ac); 1b, $\beta$-anomer of Neu5Ac; 2a, 2-deoxy 2,3-didehydro Neu5Ac (Neu5Ac2en); 2b, N-trifluoroacetylated derivative of Neu5Ac; 3, 2-deoxy- $\alpha$-Neu5Ac; 4, 4-amino-4-deoxy-NeuAc2en; 5, 4-deoxy-4-guanidinoNeu5Ac2en, now known as zanamivir; 6 , azide derivative of 4 and 5; 7, uronic acid; 8 , oseltamivir carboxylate (GS 4071); 9, peramivir; 10, a pyrrolidine, A-315675 (REF. 54); 11, oseltamivir (GS 4104); 12, divalent zanamivir.

Moreover, these X-ray structural studies identified residues within the active site that are conserved in sialidases across all influenza A and B viruses and provided an exciting opportunity for the development of compounds that should target all influenza A and B virus strains. Energetically favourable interactions between various functional groups and the residues within the binding pocket were revealed through the application of GRID software ${ }^{47}$. Most importantly, the significance of the Neu5Ac2en C-4 hydroxyl group-binding domain within the sialidase active site was realized. This realization, with further considerations, directed attention to the replacement of the Neu5Ac2en C-4 hydroxyl group by a basic group such as, in the first instance, an amino group. The 4-substituted Neu5Ac2en derivative, 4-amino-4-deoxyNeu5Ac2en (compound 4, FIG. 3), was predicted $^{39,46}$ to have higher affinity for the enzyme than the parent compound Neu5Ac2en as a result of salt-bridge formation with the conserved amino acid Glu119.

Importantly, with further analysis, it was found that the conserved Neu5Ac2en C-4 hydroxyl group-binding domain could accommodate a larger basic functional group. This analysis, together with chemical intuition, led to the conclusion that incorporation of a larger, more basic functionality in place of the Neu5Ac2en C-4 hydroxyl group was of value. Thus, substitution of 
the C-4 hydroxyl group with a guanidinyl functionality to provide 4-deoxy-4-guanidino-Neu5Ac2en (compound 5, FIG. 3) was predicted to significantly improve affinity for the enzyme. This improvement in affinity was believed to be driven by interactions between two conserved C-4 binding domain amino acids (Glu119 and Glu227) and the larger basic C-4 guanidinyl moiety ${ }^{30,39,46}$; 4-deoxy-4-guanidino-Neu5Ac2en engages most of the volume available within the sialidase active site (FIG. 5).

\section{Box 1 | Enyzmatic mechanism of influenza virus sialidase}

It was originally proposed that the solution-dominant $\alpha$-sialoside ${ }^{2} C_{5}$ conformer binds to the influenza virus sialidase and is distorted by the active-site environment from this chair conformation into an $\alpha$-boat conformer (figure). X-ray crystallographic studies ${ }^{64,65}$ of sialidase$\mathrm{Neu} 5 \mathrm{Ac}$ ( $\mathrm{N}$-acetylneuraminic acid) complexes confirmed both distortion of the substrate upon binding and the formation of a salt bridge between the substrate's negatively charged carboxyl group and highly conserved triarginyl cluster.

The departure of the aglycon residue would appear to be facilitated by the resulting conformational strain through the formation of an oxocarbocation ion intermediate, a sialosyl cation, that has been identified by kinetic isotope effect measurements and molecular modelling studies ${ }^{33,39}$. The negatively charged environment within that region of the sialidase catalytic site is thought to stabilize the charged intermediate ${ }^{33}$. A water molecule then reacts in a stereoselective manner with the sialosyl cation intermediate to afford $\alpha-\mathrm{Neu} 5 \mathrm{Ac}$ (compound 1a) as the first product of release that then mutarotates to the thermodynamically more favourable $\beta$-anomer (compound $1 \mathrm{~b}$ ) 33,39 .

Alternatively, it has been proposed ${ }^{11,66}$ that all sialidases, irrespective of origin, may trap the cation to form a glycosyl-enzyme covalent intermediate, a common feature of retaining glycohydrolases ${ }^{66}$, that is stereospecifically hydrolysed to afford compound $1 \mathrm{a}$.

Enz<smiles>O=C([O-])Oc1ccc(F)cc1</smiles>

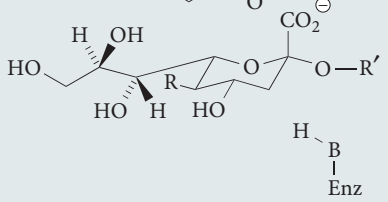

Enz $-\mathrm{R}^{\prime}$ Enz
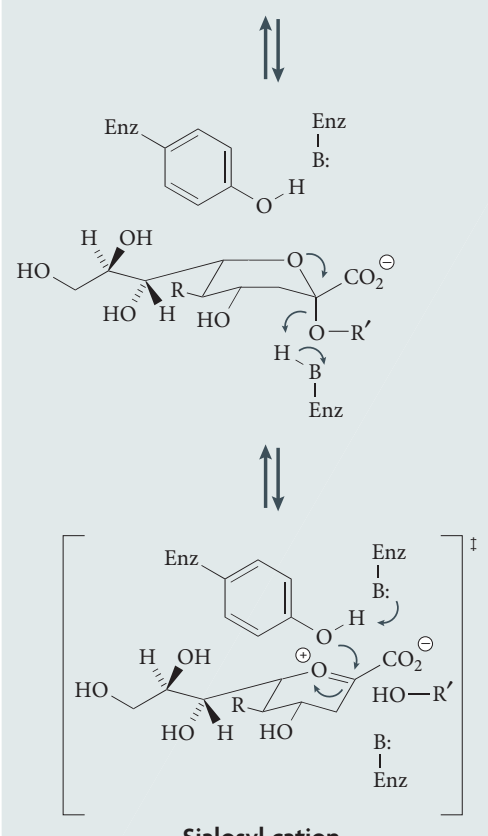

$\mathrm{R}=\mathrm{NHAC}$

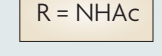

Sialosyl cation

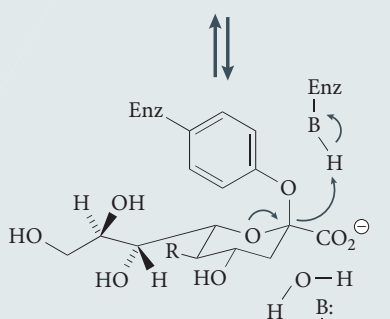

Enz

Glycosyl-enzyme intermediate<smiles>[C+]=C[CH]</smiles>

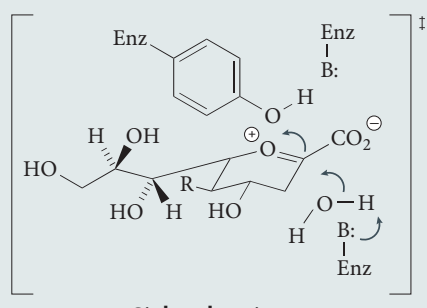

Sialosyl cation<smiles>[W]=C=[Tl]</smiles><smiles></smiles>

la

$$
\underbrace{\mathrm{HO}}_{\mathrm{HO}} \underbrace{\mathrm{OH}}_{\mathrm{HO}} \mathrm{CO}_{2}^{\ominus}
$$

lb

The two target molecules - 4-amino4-deoxy-Neu5Ac2en and 4-deoxy-4guanidino-Neu5Ac2en (FIG. 3) - were synthesized using the key C-4 azide intermediate $^{48}$ (compound 6, FIG. 3). Evaluation of these C-4 derivatives as influenza virus sialidase inhibitors confirmed that both were competitive inhibitors ${ }^{30,49}$. Moreover, both compounds were found to be highly potent inhibitors of virus replication for all influenza A and B virus strains evaluated in vitro ${ }^{30,49,50}$ and in $v i v o^{30}$. In the case of influenza A (N2) virus sialidase, 4-amino-4deoxy-Neu5Ac2en inhibited the enzyme by a factor of 100-times greater than the parent compound Neu5Ac2en (4-amino-4-deoxyNeu5Ac2en: $K_{\mathrm{i}} 4 \times 10^{-8} \mathrm{M}$; Neu5Ac2en: $\left.K_{\mathrm{i}} 4 \times 10^{-6} \mathrm{M}\right)^{49}$. As expected, the derivative 4-deoxy-4-guanidino-Neu5Ac2en was found to be more potent, with an improved affinity as much as 10,000-fold compared with Neu5Ac2en (4-deoxy-4-guanidinoNeu5Ac2en: $\left.K_{\mathrm{i}} 2 \times 10^{-10} \mathrm{M}\right)^{30,49,50}$.

$\mathrm{X}$-ray crystallographic structure determination of influenza virus sialidase-inhibitor complexes for 4-amino-4-deoxy-Neu5Ac2en and 4-deoxy-4-guanidino-Neu5Ac2en confirmed, in general, that these inhibitors engaged the enzyme's active site in the predicted binding modes $^{30}$. Specifically, the 4-amino group of 4-amino-4-deoxyNeu5Ac2en was shown ${ }^{30}$ to establish a salt bridge with Glu119, and although 4-deoxy-4-guanidino-Neu5Ac2en displayed the predicted ${ }^{30,46}$ lateral binding between the terminal guanidinyl nitrogens and the carboxylate of Glu227, Glu119 was found to be slightly further removed ${ }^{30}$ than proposed and stacked parallel to the guanidinyl group $^{38}$. However, Glu119 is still within a distance close enough for electrostatic interaction with the guanidinyl group ${ }^{30,38}$.

Fortuitously, 4-deoxy-4-guanidinoNeu5Ac2en was found to be highly selective for influenza virus sialidase and displayed considerably lower affinity for other sialidases from different sources ${ }^{49}$. The selectivity of influenza virus sialidase inhibitors has become of increasing interest as a result of potential side effects in patients as a result of, in part, the possible inhibition of endogenous human sialidases ${ }^{51}$.

The more potent inhibitor, 4-deoxy-4guanidino-Neu5Ac2en (compound 5, FIG. 3), was selected as the lead drug candidate by Glaxo under the generic name zanamivir. Owing to its limited oral bioavailability (due to its highly polar nature and rapid excretion of the compound), it was developed as an inhaled formulation, 
which delivers the drug directly to the primary site of infection. As zanamivir was a first-in-class drug, significant effort in the establishment of appropriate end-point measurements of clinical benefit, in cooperation with regulatory agencies such as the US Food and Drug Administration (FDA), was needed. Following its success in clinical trials, zanamivir was approved in 1999 as the first sialidase-targeting anti-influenza drug, with the tradename Relenza.

Discovery and development of oseltamivir. The discovery of zanamivir provided a platform for further sialidase-targeted antiinfluenza drugs. Significant work has been undertaken in structure-activity relationship studies with Neu5Ac-based derivatives and uronic acid carbohydrate-based templates derived from $\mathrm{N}$-acetylglucosamine

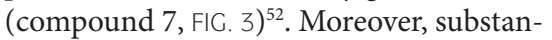
tial effort has been applied towards the development of influenza virus sialidase inhibitors that are based on non-carbohydrate templates (for a review, see REF. 11). For example, potent and selective inhibitors of influenza virus sialidase and of influenza virus infection in vivo have been developed based on a range of core templates including cyclohexenes such as oseltamivir carboxylate (compound 8, FIG. 3; originally known as GS 4071) ${ }^{31}$; cyclopentanes such as peramivir ${ }^{53}$ (compound 9, FIG. 3 ); and pyrrolidines such as A-315675 (compound 10, FIG. 3) $)^{54}$. Most noteworthy has been the development of the cyclohexene derivative GS 4071, and its prodrug, oseltamivir, the first orally active sialidase inhibitor to be approved for treating influenza.

Significant synthetic organic and medicinal chemistry based on the functionalized cyclohexene and cyclohexane shikimic acid and quinic acid core templates, respectively, were undertaken in the discovery of GS 4071. On the basis of interactions of the unsaturated Neu5Ac derivatives 4 and 5, three key concepts were used in the discovery of GS 4071 and oseltamivir. First, based on previous mechanistic studies ${ }^{33,36,39}$, positioning the double bond in the inhibitor to more closely mimic the putative transition state sialosyl cation (BOX 1) was investigated.

Second, replacing the glycerol moiety of 4-amino-4-deoxy-Neu5Ac2en by a lipophilic group was explored on the basis that the hydrophobic backbone of the glycerol side chain makes contact with the protein, even though the C-8 and C-9 hydroxyl groups make a bidentate interaction with Glu276. This replacement was attempted in the hope a
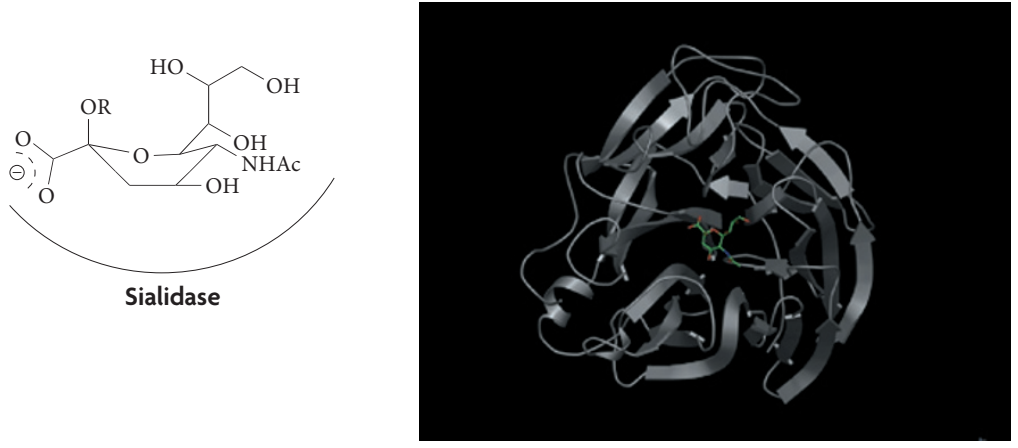

b<smiles>CC(=O)N[C@H]1[C@H](O)C[C@@](O)(C(=O)O)O[C@H]1[C@H](O)[C@H](O)CO</smiles>
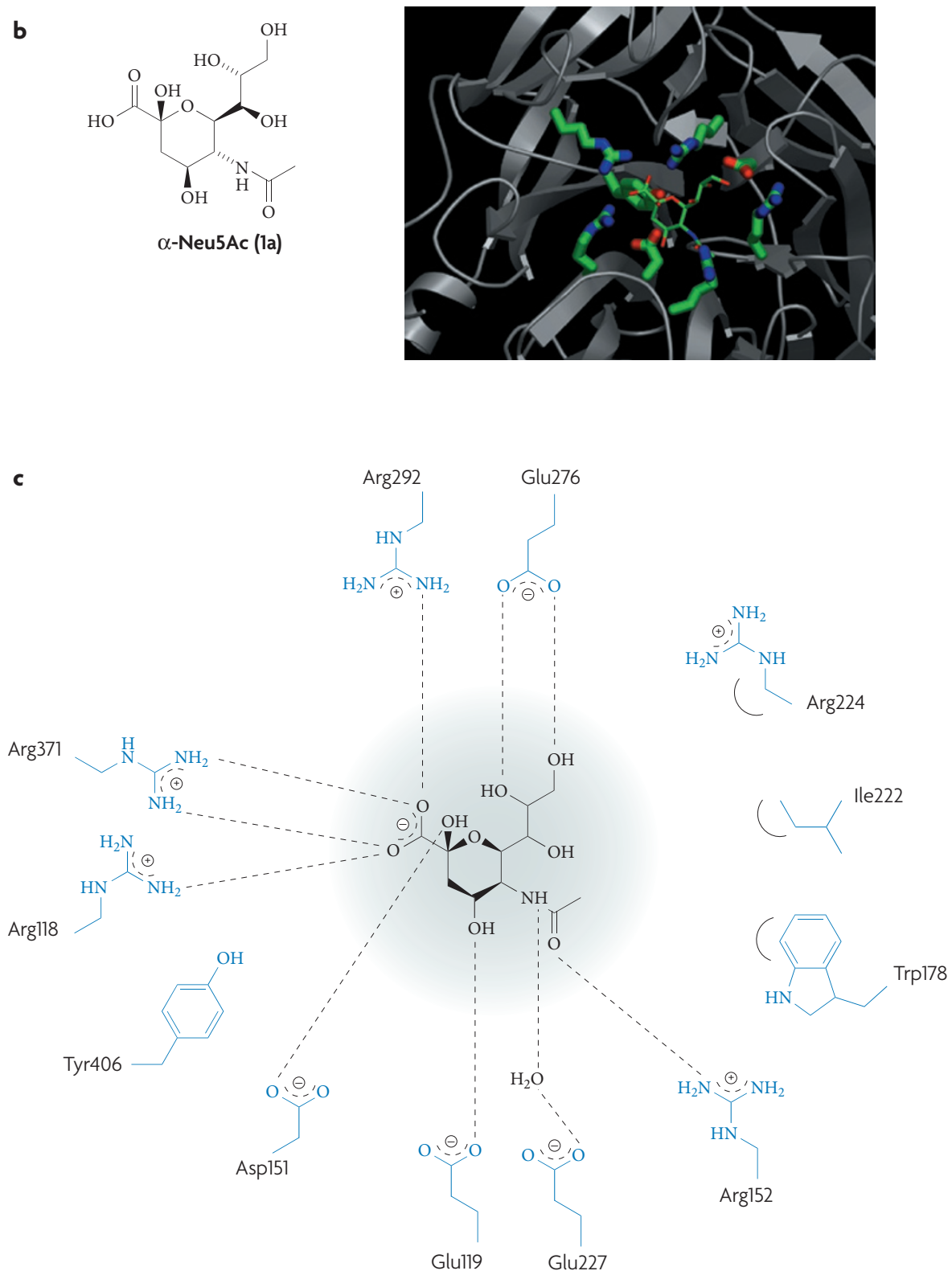

Figure 4 | Interaction of $\alpha-N$-acetylneuraminic acid (1a) with influenza virus sialidase. a $\alpha-N$-acetylneuraminic acid ( $\alpha-N e u 5 A c)$ bound in a boat-like conformation to the influenza virus sialidase active site. $\mathbf{b}$ | Eight invariant amino-acid residues make direct contact with Neu5Ac. c | Neu5Ac interactions with the influenza A virus sialidase active site (derived from LIGPLOT software ${ }^{67}$ ). 
that optimization of the hydrophobic character would lead to new sialidase inhibitors with improved lipophilicity while maintaining inhibitor activity. Wholesale replacement of the glycerol side chain by a lipophilic moiety was counter-intuitive, as it had been demonstrated (see previous section) that the

glycerol-binding domain engaged the $\mathrm{C}-8$ and C-9 hydroxyl groups through specific interactions (FIG. 4C).

Extensive manipulation of the alkyl side chain, in an elegant structure-activity investigation, led to the development of the optimized 3-pentyl ether side chain. An

a
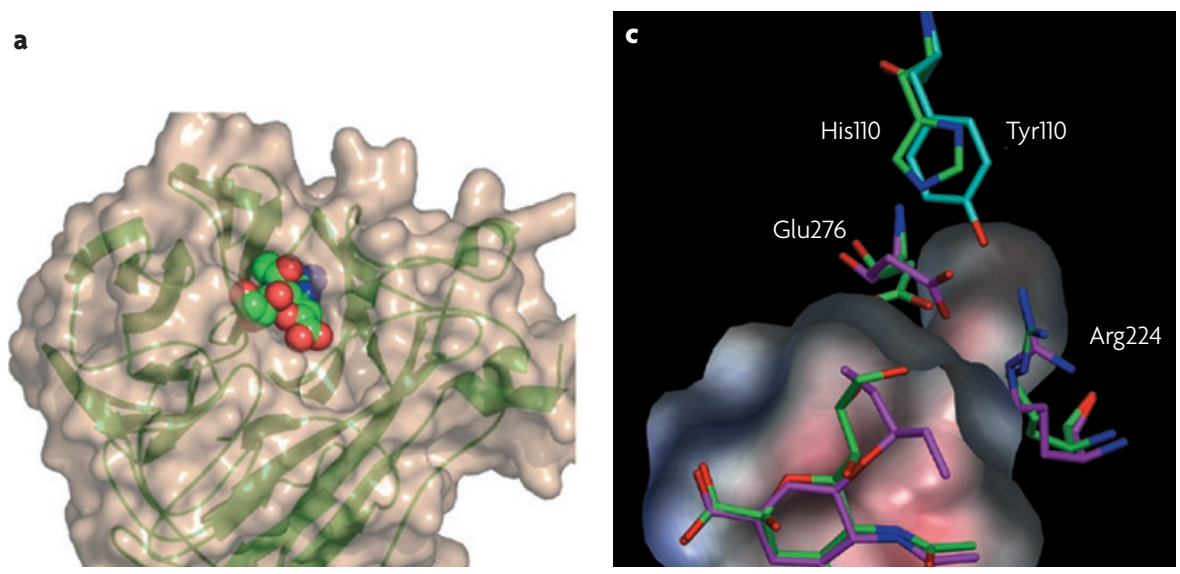

b
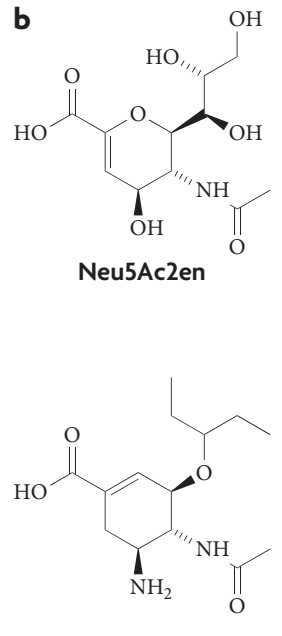

Oseltamivir (free acid)

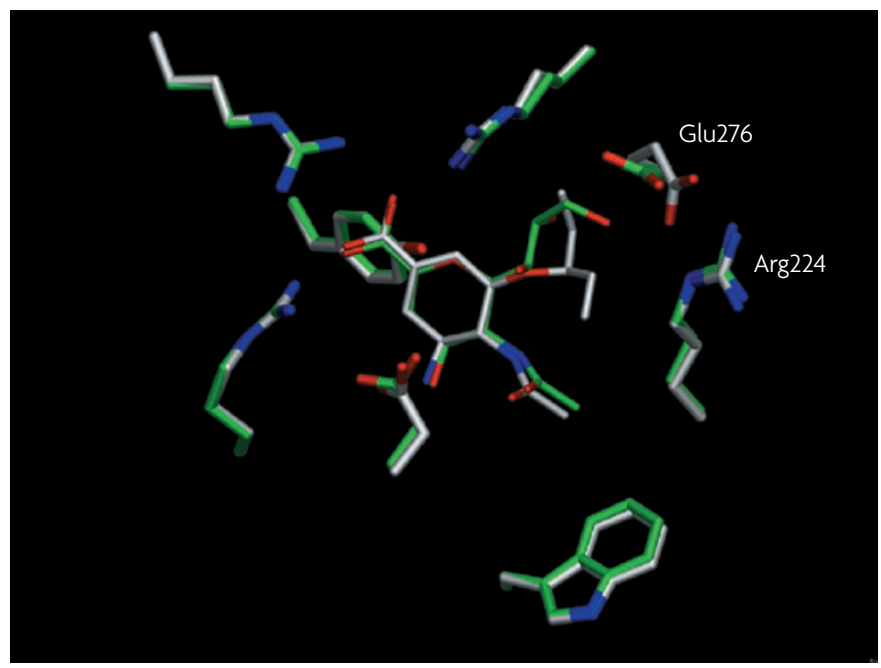

Figure 5 | Structures of influenza virus sialidase with zanamivir and oseltamivir carboxylate. a | The inhibitor zanamivir is shown in CPK model in complex with influenza virus $A$ sialidase. b Oseltamivir carboxylate engages the sialidase active site via an induced fit mechanism. The crystal structure of the influenza virus sialidase-oseltamivir carboxylate complex has provided an excellent rationale of why this compound can efficiently engage the sialidase active site ${ }^{11,31,55}$. Two superimposed structures are shown. In green is the X-ray crystal structure of influenza virus sialidase selected activesite amino-acid residues in complex with 2-deoxy 2,3-didehydro-N-acetylneuraminic acid (Neu5Ac2en; compound 2a, FIG. 3) In white is the X-ray crystal structure of influenza virus sialidase with the same selected active-site amino-acid residues in complex with oseltamivir carboxylate (compound 8, FIG. 3). The cyclohexene ring of oseltamivir carboxylate (white) is oriented within the sialidase active site such that the carboxylate, acetamide and C-4 substituent interact with the sialidase active site similar to those observed for Neu5Ac2en (green) and derivatives, as expected. However, complexation of oseltamivir carboxylate induces Glu276 to adopt an alternative conformation that is stabilized by a charge-charge interaction to Arg224. This induced fit establishes a hydrophobic area in which the two ethyl groups of oseltamivir carboxylate are then well accommodated. c | Surface display of $N$-acetylneuraminic acid (atomic colours) and oseltamivir carboxylate (carbon atoms in magenta) influenza virus A sialidase structures superimposed. The key amino-acid residue Glu276 that undergoes reorientation upon binding of oseltamivir carboxylate (see main text) and Arg224 are shown. The His110 (atomic colours) to Tyr110 (cyan) mutation results in oseltamivir resistance, as it seems to prevent the essential rearrangement of Glu276. These mutants remain sensitive to zanamivir as this inhibitor engages Glu276 in an identical manner to that of the substrates recognized by the enzyme (FIG. 4).
X-ray crystallographic study of an influenza virus sialidase-GS 4071 complex clearly showed that the architecture of the active site had been altered on binding of GS 4071 (REFS 31,55). Specifically, Glu276 reorients outwards from the glycerol side-chain binding domain to interact with Arg224 and in doing so generates a considerable hydrophobic area within this domain. This rearrangement, which was not predictable, provided exciting new opportunities for further sialidase inhibitor-based anti-influenza drug discovery. Although GS 4071 binds in an identical fashion to zanamivir and other Neu5Ac2en derivatives, this induced fit is essential for the inhibitor to successfully engage the active site to provide the inhibitor's potent efficacy (FIG. 5 c).

Third, although it had been hoped that GS 4071 (which is more lipophilic than zanamivir) might have had sufficient oral bioavailability, this was found not to be the case, and so a prodrug strategy was used. Oseltamivir (GS 4104; compound 11, FIG. 3), the ethyl ester prodrug of GS 4071, is readily converted to the active form in vivo by the action of endogenous esterases. Following its success in clinical trials as an orally administered treatment for influenza virus infection, oseltamivir (developed by Gilead) was approved in late 1999, and is now marketed by Roche under the trade name Tamiflu.

\section{Experience with sialidase inhibitors}

As zanamivir and then oseltamivir came to market, the questions of clinically significant resistance development and possible unknown side effects remained to be answered. It was hoped that zanamivir, a drug derived from the naturally occurring sialic acid Neu5Ac with minimal additional functionalization, would not result in viable mutants - as functionally important amino-acid residues had been targeted - or unknown side effects. So far, over 8 years of clinical experience with zanamivir has not provided evidence to suggest otherwise, although this may simply be the result of the limited use of this drug.

In the case of oseltamivir, which has been much more widely used, a viable resistant influenza virus mutant has emerged ${ }^{56}$. Interestingly, this mutation targets and effectively blocks the essential rearrangement of Glu276 within the sialidase active site and as a consequence the drug has significantly reduced affinity (FIC. 5b). This oseltamivirresistant virus remains sensitive to zanami$v^{5}{ }^{56}$. These experiences may provide some evidence that maintaining a strong resemblance to the natural substrate, Neu5Ac, might 
reduce the prospect of the development of viable drug-resistant mutants. This is because when drug binding depends on either activesite amino-acid reorientation or interactions with non-essential active-site amino acids, the possibility of escape mutants might increase. With regards to adverse effects of sialidase inhibitors in general, the only significant adverse effects that have been reported in the past 2 years have been some speculation about adverse neuropsychiatric effects of oseltamivir in certain age groups ${ }^{57}$.

\section{Outlook}

In light of the current pandemic threat and the emergence of resistance to oseltamivir, the development of next-generation antiinfluenza drugs must be a high priority. To this end, the US FDA has established a fast-track programme for the development of such drugs. So far, the FDA has provided fast-track designation for the Biocryst injectable candidate peramivir (compound 9, FIG. 3). Biota and Sankyo have also announced their intention to develop a new influenza virus sialidase inhibitor that is a divalent zanamivir (compound 12, FIG. 3), which is currently in clinical trials.

Both of these developments are important as they could give rise to alternatives to the currently available drugs that may be required in the case of a pandemic or with the appearance of significant drug resistance. An injectable drug such as peramivir might be of great value to patients who cannot readily take tablets or have limited lung capacity. Also, a long-acting sialidase inhibitor, such as divalent zanamivir, that might reduce the number of treatments required to as little as once a week compared with the current twice-daily requirement is appealing.

A further important consideration for the development of next-generation sialidase inhibitors is the cost of drug production. The experiences from the development of both the relatively expensive zanamivir and oseltamivir provide valuable insight towards the design of next-generation potent influenza virus sialidase inhibitors from relatively inexpensive starting materials, such as $N$-acetylglucosamine ${ }^{52}$.

Recently, Russell and colleagues have described that influenza virus sialidases may be grouped into two distinct families: namely Group 1 and Group 2 influenza virus sialidases $^{58}$. It has been suggested that the Group 1 sialidases such as N1 from the now infamous avian H5N1 strain undergo significant rearrangement around the so-called 150 loop upon binding of substrates and inhibitors. In apo structures of the Group 1 influenza virus sialidases, the 150 loop is in a more open orientation that presents a larger active-site cavity. These enzymes complexed with the inhibitor oseltamivir carboxylate reveal that this 150 loop eventually closes to tightly coordinate the inhibitor ${ }^{58}$. The fact that there is a more open enzyme architecture in the apo and initially inhibitor-complexed structures provides new and exciting opportunities for exploitation in sialidasetargeted anti-influenza drug discovery.

An important remaining question is who will fund the development of such compounds. It is unlikely that pharmaceutical companies would race to bring to market another anti-influenza drug, particularly as there are already two good drugs available. It may be that national governments or notfor-profit organizations will have to support such development, particularly if nations wish to stockpile a variety of anti-influenza drugs.

Alternative drug discovery targets ${ }^{59-61}$, such as the RNA polymerase ${ }^{59}$, the haemagglutinin protein $^{60}$ or the $\mathrm{M} 2$ ion channel protein $^{61}$, that are essential in the virus' life cycle are also under investigation and may, in either a combination therapy approach ${ }^{61}$ with the sialidase inhibitors or in their own right, provide new classes of anti-influenza drugs. Combination therapy might also reduce the potential of resistance development ${ }^{61}$. Of course, although anti-influenza drugs will buy time and save lives, the 'Holy Grail' would be the development of a universal vaccine that would protect against all influenza virus strains past, present and future. Much effort is going on in the development of improved vaccines; however, there is still a long way to $\mathrm{go}^{62,63}$. There is no doubt that we must continue efforts in drug discovery to win the war against this deadly virus, or risk another potential pandemic that may result in mortality similar to that experienced between 1918 and 1919.

Mark von Itzstein is at the Institute for Glycomics, Gold Coast Campus, Griffith University, Queensland 4222, Australia e-mail:m.vonitzstein@griffith.edu.au

doi: $10.1038 / \mathrm{nrd} 2400$

1. Yuen, K. Y. \& Wong, S. S. Human infection by avian influenza A H5N 1. Hong Kong Med. J. 11, 189-199 (2005)

2. Maines, T. R. et al. Avian influenza (H5N1) viruses isolated from humans in Asia in 2004 exhibit increased virulence in mammals. J. Virol. 79, 11788-11800 (2005).

3. Buchy, P. et al. Influenza A/H5N 1 virus infection in humans in Cambodia. J. Clin. Virol. 39, 164-168 (2007).

4. Douglas, R. G. Jr. Prophylaxis and treatment of influenza. N. Engl. J. Med. 322, 443-450 (1990).
Wintermeyer, S. M. \& Nahata, M. C. Rimantadine: a clinical perspective. Ann. Pharmacother. 29, 299-310 (1995)

6. Pinto, L. H., Holsinger, L. J. \& Lamb, R. A. Influenza virus M2 protein has ion channel activity. Cell 69, 517-528 (1992).

7. Hay, A. J. et al. in Options for the Control of Influenza Virus II (ed. Hannoun, C. E.) 281-288 (Excerpta Medica, Amsterdam, 1993).

8. Hayden, F. G. in Principles and Practice of Infectious Disease (eds Mandell, G. L., Douglas, R. G. J. $\delta$ Bennett, J. E.) 3-15 (Churchill Livingston, New York, 1993)

9. Laver, G. \& Garman, E. Pandemic influenza: its origin and control. Microbes Infect. 4, 1309-1316 (2002).

10. Skehel, J. J. \& Wiley, D. C. Receptor binding and membrane fusion in virus entry: the influenza hemagglutinin. Annu. Rev. Biochem. 69, 531-569 (2000).

11. Rich, J. R., Gehle, D. \& von Itzstein, M. in Comprehensive Glycoscience: from Chemistry to Systems Biology (eds. Kamerling, J. et al) (Elsevier, Amsterdam, 2007).

12. Webster, R. G. et al. in Options for the Control of Influenza Virus II (ed. Hannoun, C.) 177-185 (Excerpta Medica, Amsterdam, 1993).

13. Wagner, R., Matrosovich, M. \& Klenk, H.-D Functional balance between haemagglutinin and neuraminidase in influenza virus infections. Rev. Med. Virol. 12, 159-166 (2002).

14. Oxford, J. S. Influenza A pandemics of the 20th century with special reference to 1918: virology, pathology and epidemiology. Rev. Med. Virol. 10 119-133 (2000)

15. Herrler, G., Hausmann, J. \& Klenk, H.-D. in Biology of the Sialic Acids (ed. Rosenberg, A.) 315-336 (Plenum Press, New York, 1995)

16. Couceiro, J. N., Paulson, J. C. \& Baum, L. G. Influenza virus strains selectively recognize sialyloligosaccharides on human respiratory epithelium; the role of the host cell in selection of hemagglutinin receptor specificity. Virus Res. 29, 155-165 (1993)

17. Suzuki, Y. et al. Sialic acid species as a determinant of the host range of influenza A viruses. J. Virol. 74 11825-11831 (2000).

18. Matrosovich, M. \& Klenk, H.-D. Natural and synthetic sialic acid-containing inhibitors of influenza virus receptor binding. Rev. Med. Virol. 13, 85-97 (2003).

19. Wilson, I. A., Skehel, J. J. \& Wiley, D. C. Structure of the haemagglutinin membrane glycoprotein of influenza virus at 3 Å resolution. Nature 289 366-373 (1981).

20. Weis, W. et al. Structure of the influenza virus haemagglutinin complexed with its receptor, sialic acid. Nature 333, 426-431 (1988)

21. Sauter, N. K. et al. Crystallographic detection of a second ligand binding site in influenza virus hemagglutinin. Proc. Natl Acad. Sci. USA 89, 324-328 (1992).

22. Watowich, S. J., Skehel, J. J. \& Wiley, D. C. Crystal structures of influenza virus hemagglutinin in complex with high-affinity receptor analogs. Structure 2, 719-731 (1994).

23. Colman, P. M. \& Ward, C. W. Structure and diversity of influenza virus neuraminidase. Curr. Top. Microbiol. Immunol. 114, 177-255 (1985).

24. Palese, P., Tobita, K., Ueda, M. \& Compans, R. W. Characterization of temperature sensitive influenza virus mutants defective in neuraminidase. Virology 61 , 397-410 (1974)

25. Liu, C., Eichelberger, M. C., Compans, R. W. \& Air, G. M. Influenza type $A$ virus neuraminidase does not play a role in viral entry, replication, assembly, or budding. J. Virol. 69, 1099-1106 (1995).

26. Varghese, J. N., Laver, W. G. \& Colman P. M. Structure of the influenza virus glycoprotein antigen neuraminidase at $2.9 \AA$ resolution. Nature $303,35-40$ (1983).

27. Colman, P. M., Varghese, J. N. \& Laver, W. G. Structure of the catalytic and antigenic sites in influenza virus neuraminidase. Nature 303, 41-44 (1983).

28. Smith, B. J. et al. Structural studies of the resistance of influenza virus neuraminidase to inhibitors. J. Med. Chem. 45, 2207-2212 (2002).

29. Colman, P. M. Neuraminidase inhibitors as antivirals. Vaccine 20, S55-S58 (2002).

30. von Itzstein, M. et al. Rational design of potent sialidase-based inhibitors of influenza virus replication. Nature 363, 418-423 (1993). 
31. Kim, C. U. et al. Influenza neuraminidase inhibitors possessing a novel hydrophobic interaction in the enzyme active site: design, synthesis, and structural analysis of carbocyclic sialic acid analogues with potent anti-influenza activity. J. Am. Chem. Soc. 119, 681-690 (1997)

32. Lentz, M. R., Webster, R. G. \& Air, G. M. Site-directed mutation of the active site of influenza neuraminidase and implications for the catalytic mechanism. Biochemistry 26, 5351-5358 (1987).

33 Chong A. K. Pegg M. S. Taylor, N. R. \& von Itzstein, M. Evidence for a sialosyl cation transitionstate complex in the reaction of sialidase from influenza virus. Eur. J. Biochem. 207, 335-343 (1992).

34. Tiralongo, J., Pegg, M. S. \& von Itzstein, M. Effect of substrate aglycon on enzyme mechanism in the reaction of sialidase from influenza virus. FEBS Lett. 372, 148-150 (1995).

35. Drzeniek, R. Viral and bacterial neuraminidases. Curr. Top. Microbiol. Immunol. 59, 35-74 (1972).

36. Flashner, M. Kessler, J. \& Tanenbaum, S. W. The interaction of substrate-related ketals with bacterial and viral neuraminidases. Arch. Biochem. Biophys. 221, 188-196 (1983).

37 Colman, P. M. Influenza virus neuraminidase: structure, antibodies, and inhibitors. Protein Sci. 3 , 1687-1696 (1994).

38. Varghese, J. N., Epa, V. C. \& Colman, P. M Three-dimensional structure of the complex of 4-guanidino-Neu5Ac2en and influenza virus neuraminidase. Protein Sci. 4, 1081-1087 (1995).

39. Taylor, N. R. \& von Itzstein, M. Molecular modeling studies on ligand binding to sialidase from influenza virus and the mechanism of catalysis. J. Med. Chem 37, 616-624 (1994).

40. Wilson, J. C. \& von Itzstein, M. Recent strategies in the search for new anti-influenza therapies. Curr. Drug Targets 4, 389-408 (2003).

41. Colman, P. M. et al. Preparation of 2-deoxy- $N$ acetylneuraminic acid derivatives as antiviral compounds which bind the active site of influenza neuraminidase. Patent WO9206691 (1992)

42. Palese, P., Schulman, J. L., Bodo, G. \& Meindl, P. Inhibition of influenza and parainfluenza virus replication in tissue culture by 2-deoxy-2,3-dehydro- $N$ trifluoroacetylneuraminic acid (FANA). Virology 59 490-498 (1974)

43. Palese, P. \& Compans, R. W. Inhibition of influenza virus replication in tissue culture by 2-deoxy-2,3-dehydro- $N$ trifluoroacetylneuraminic acid (FANA): mechanism of action. J. Gen. Virol. 33, 159-163 (1976).

44. Palese, P. \& Schulman, J. L. in Chemoprophylaxis and Viral Infections of the Respiratory Tract (ed. Oxford, J. S.) 189-205 (CRC Press, Cleveland, 1977)
45. Nöhle, U., Beau, J. M. \& Schauer, R. Uptake, metabolism and excretion of orally and intravenously administered, double-labeled $N$-glycoloylneuraminic acid and single-labeled 2-deoxy-2,3-dehydro- $\mathrm{N}$ acetylneuraminic acid in mouse and rat. Eur. J. Biochem. 126, 543-548 (1982).

46. von Itzstein, M. et al. A study of the active site of influenza virus sialidase: an approach to the rational design of novel anti-influenza drugs. J. Med. Chem. 39, 388-391 (1996).

47. Goodford, P. J. A computational procedure for determining energetically favorable binding sites on biologically important macromolecules. J. Med. Chem. 28, 849-857 (1985).

48. von Itzstein, M., Wu, W.-Y. \& Jin, B. The synthesis of 2,3-didehydro-2,4-dideoxy-4-guanidinyl- $N$ acetylneuraminic acid: a potent influenza virus sialidase inhibitor. Carbohydr. Res. 259, 301-305 (1994).

49. Holzer, C. T. et al Inhibition of sialidases from viral, bacterial and mammalian sources by analogues of 2-deoxy-2,3-didehydro- $N$-acetylneuraminic acid modified at the C-4 position. Glycoconj. J. 10, 40-44 (1993).

50. Woods, J. M. et al. 4-Guanidino-2,4-dideoxy-2,3dehydro- $N$-acetylneuraminic acid is a highly effective inhibitor both of the sialidase (neuraminidase) and of growth of a wide range of influenza A and B viruses in vitro. Antimicrob. Agents Chemother. 37 1473-1479 (1993).

51. Li, C. Y. et al. A nonsynonymous SNP in human cytosolic sialidase in a small Asian population results in reduced enzyme activity: potential link with severe adverse reactions to oseltamivir. Cell Res. 17, 357-362 (2007).

52. Mann, M. C. et al. Unsaturated N-acetyl-Dglucosaminuronic acid glycosides as inhibitors of influenza virus sialidase. Glycoconj. J. 23, 127-133 (2006)

53. Babu, Y. S. et al. BCX-1812 (RWJ-270201): discovery of a novel, highly potent, orally active, and selective influenza neuraminidase inhibitor through structurebased drug design. J. Med. Chem. 43, 3482-3486 (2000)

54. DeGoey, D. A. et al. Enantioselective synthesis of antiinfluenza compound A-315675. J. Org. Chem. 67 5445-5453 (2002)

55. Kim, C. U. et al. Structure-activity relationship studies of novel carbocyclic influenza neuraminidase inhibitors. J. Med. Chem. 41, 2451-2460 (1998).

56. Le, Q. M. et al. Avian flu: isolation of drug-resistant H5N 1 virus. Nature 437, 1108 (2005).

57. Okumura, A. et al. Oseltamivir and delirious behavior in children with influenza. Pediatr. Infect. Dis. J. 25 572 (2006)
58. Russell, R. J., et al The structure of $\mathrm{H} 5 \mathrm{~N} 1$ avian influenza neuraminidase suggests new opportunities for drug design. Nature 443, 45-49 (2006).

59. Tsai, C. H., Lee, P. Y., Stollar, V., Li, M. L. Antiviral therapy targeting viral polymerase. Curr. Pharm. Des. 12, 1339-1355 (2006)

60. De Clercq, E. \& Neyts, J. Avian influenza A (H5N1) infection: targets and strategies for chemotherapeutic intervention. Trends Pharmacol. Sci. 28, 280-285 (2007).

61. Ilyushina, N. A. Bovin, N. V., Webster, R. G. $\delta$ Govorkova, E. A. Combination chemotherapy, a potential strategy for reducing the emergence of drug-resistant influenza A variants. Antiviral Res. 70, 121-131 (2006)

62. Hasegawa, H. et al. Development of a mucosal vaccine for influenza viruses: preparation for a potential influenza pandemic. Expert Rev. Vaccines 6, 193-201 (2007).

63. Carrat, F. \& Flahault, A. Influenza vaccine: the challenge of antigenic drift. Vaccine 25, 6852-6862 (2007).

64. Varghese, J. N., McKimm-Breschkin, J. L., Caldwell, J. B., Kortt, A. A. \& Colman P. M. The structure of the complex between influenza virus neuraminidase and sialic acid, the viral receptor. Proteins 14, 327-332 (1992).

65. Burmeister, W. P. et al. Influenza B virus neuraminidase can synthesize its own inhibitor. Structure 1, 19-26 (1993).

66. Zechel, D. L. \& Withers, S. G. Dissection of nucleophilic and acid-base catalysis in glycosidases. Curr. Opin. Chem. Biol. 5, 643-649 (2001).

67. Wallace, A. C., Laskowski, R. A. \& Thornton, J. M. LIGPLOT: a program to generate schematic diagrams of protein-ligand interactions. Protein Eng. 8, 127-134 (1995).

\section{Acknowledgements}

The Australian Research Council is acknowledged for its generous support through the award of a Federation Fellowship. J. Dyason is thanked for providing a number of the computergenerated graphics and for reading this manuscript.

DATABASES

Swissprot ENZYME: http://ca.expasy.org/enzyme/ Exo- $\alpha$-sialidase

FURTHER INFORMATION

Griffith University Institute of Glycomics:

http://www.qriffith.edu.au/centre/glycomics

World Health Organization: www.who.int/csr/disease/

avian influenza/country/cases table $2007 \quad 07 \quad 25 /$

ALL LINKS ARE ACTIVE IN THE ONLINE PDF 